\title{
Dynamics of population decoding with strong inhibition
}

\author{
Thomas Trappenberg ${ }^{12}$ \\ 1 Dalhousie University, Canada \\ 2 Bernstein Center for Computational Neuroscience Freiburg, Germany
}

\begin{abstract}
Decoding information from a population of noisy neurons can be achieved efficiently with center-surround recurrent networks. Here we study such networks with continuing external input and investigate the dynamics of decoding with varying inhibition strength in the network. We find that the best decoding is achieved at the onset of the memory regime in such networks.
\end{abstract}

\section{Introduction}

In the brain, information is typically represented by a population of neurons. For example, while individual neurons in the primary visual cortex respond maximally to specific orientations of line segments, they also respond to a lesser extend to neighboring orientations. Thus, even with a 'pure' image there are many neurons that respond. This is important as such states are likely to represent probabilities of specific events [1]. Furthermore, cortical neurons are very noisy and their firing rates are low so that decoding the information from a population becomes crucial for reliable information processing in the brain.

Mathematically it is well established how to decode information in specific situations [2]. For example, if we consider Gaussian tuning curves we could fit a Gaussian through the data points given by the neuron response to achieve a maximum likelihood (ML) estimate of the stimulus which is optimal with these assumptions. While optimal decoding is of some theoretical and practical interest, much more important for brain processing is its efficient implementation. Several researcher have pointed out that ML estimation in the above mentioned case can be achieved with recurrent networks [3,4]. This is significant as these networks enable fast computation consistent with the functional mechanisms of the cortex as captured by neural fields theory [5-7]. Furthermore, while the optimality can be proven in some cases [4], optimality in a statistical sense is not fundamentally required. Instead, it is possible that this is the principle decoding mechanism in the brain and this mechanism should therefore be studied in more general circumstances, such as in the case of multiple inputs.

Decoding mechanisms with neural fields have been studied mainly in a model where noisy input was applied as initial states of the network and where the asymptotic state of sustained activity packets (bubbles) are used for optimal decoding. In this paper we explore the dynamics of decoding when input is sustained for some time, resembling more closely transient input in the brain. We then explore the regime with strong lateral inhibition and show that this regime is well suited for fast population decoding. 


\section{Population decoding with recurrent networks}

We consider a standard recurrent rate neural network model with $N$ nodes in which the time evolution of the internal state $u_{i}$ is given by

$$
\tau \frac{\mathrm{d} u_{i}(t)}{\mathrm{d} t}=-u_{i}(t)+\sum_{j} w_{i j} r_{j}(t) \Delta x+I_{i}^{\mathrm{ext}}(t),
$$

where $\tau$ is a time constant, $I_{i}^{\text {ext }}$ is the external input applied to the network and $\Delta x=$ $2 \pi / N$ is a scale factor. The rate $r_{i}$ is related to $u_{i}$ by a sigmoidal gain function $g(u)=$ $1 /(1+\exp (-\beta u))$ with a slope parameter $\beta=0.1$. Applications of this model to population decoding commonly use a gain function with divisive inhibition [8], but the principal findings reported here do not depend critically on the form of the gain function. The weight matrix, w, describes center-surround interactions in the network with a shifted Gaussian profile,

$$
w_{i j}=A_{\mathrm{w}}\left(\mathrm{e}^{-((i-j) * \Delta x)^{2} / 2 \sigma_{w}^{2}}-C\right) .
$$

This dynamic model exhibits several regimes characterized by different possible asymptotic states [7]. If the inhibition, $C$, is low compared to excitation in the network, then the excitation will spread through the network resulting in runaway activity. In contrast, if inhibition is dominating, then any activity in the field will decay without external reinforcement. In an intermediate regime it is possible to have activity packets where localized activity is stable. We call this mode of the model the memory regime.

The later two regimes are depicted in Figure 1 in the context of population decoding. In these experiments we supply a static noisy input to the field over 20 timesteps. This input was chosen as a Gaussian around the middle node $\left(x_{0}=50\right)$ with additive white noise of strength $n_{\eta}=0.5$,

$$
I_{\text {ext }}=I_{0}+A\left(\mathrm{e}^{-((i-j) * \Delta x)^{2} / 2 \sigma^{2}}+n_{\eta} \eta\right.
$$

where $I_{0}$ is a background field and $\eta$ is a normal distributed random number. When an inhibition constant of $c=0.05$ is used, the field developed into a clean bubble around the middle node after the input was removed at time $t=20$, demonstrating perfect decoding. The sustained localized activity in the neural field without external input demonstrates the above mentioned memory regime.

Traces of the noisy input are not apparent in the $c=0.05$ case, and it seems one has to wait until the bubble forms to perform accurate decoding. This is different when running the same simulation with larger inhibition. Figure 1 shows the case for $c=$ 0.07 . Traces of the noisy input are now also visible during the time the external input is supplied, which is partly enhanced by the fact that a smaller range of values is depicted by the gray scale in the figure. The inhibition is now too large to sustain an activity packet after input is removed. However, the increased competition facilitates a cleaning of the signal even during the time when the signal is applied so that some form of population decoding is supported. While this might be less accurate than in the previous case, an advantage would certainly be that the decoding can be achieved much earlier. This assertion is investigated in the next section. 

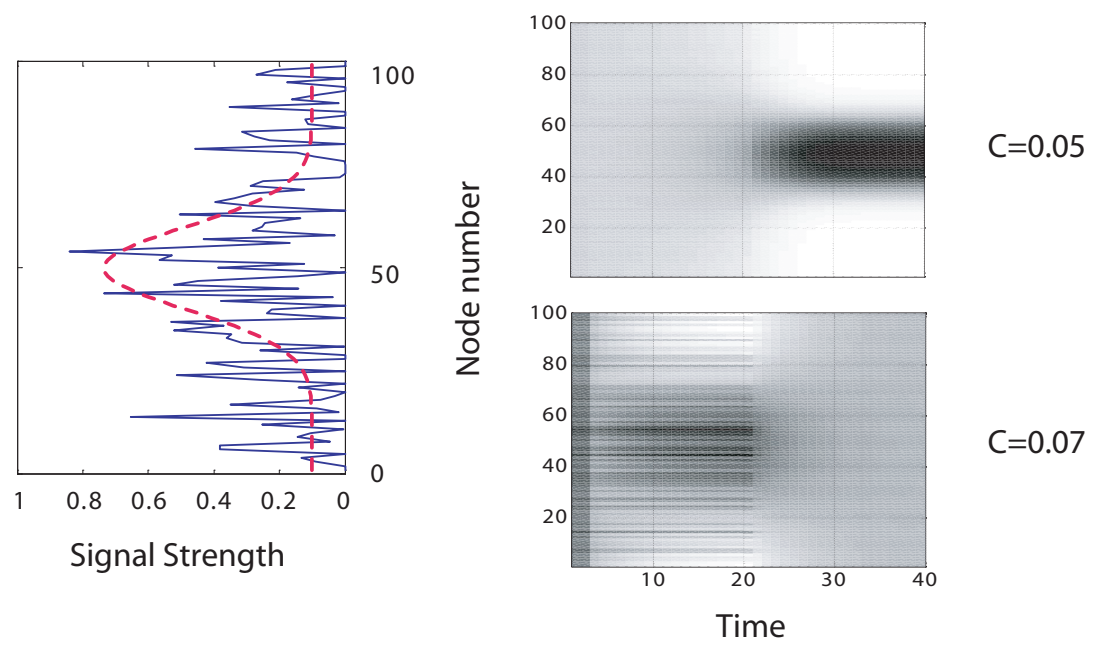

Fig. 1. Noisy population decoding with weak and strong inhibition in neural fields. The noisy input derived from the dashed line on the left is applied until $t=20$ to the neural fields shown on the right

\section{Quality of decoding with varying inhibition over time}

To assess the quality of decoding with time and different inhibition constants we ran decoding experiments over 100 trials in each condition. While we have used signals with static noise in Figure 1, we report now on the results when changing the noise after each unit of time, simulating ongoing fluctuations in the input signal over time. This represents a more plausible implementation of decoding conditions in the brain although we found similar results in the static noise case.

To assess the decoding quality we use a center of mass scheme to determine the prediction of the feature value encoded in the neural field. This was done for the original input signal and at each time step during the dynamic decoding in the recurrent network. An example trace of the decoding error is shown in Figure 2A. For the shown strength of inhibition, the decoding error continuously decreases even while external input is supplied. The decoding error only improves slightly after the external input is removed at $t=20$. The decoding error increases with increasing noise level as shown in Figure $2 \mathrm{~B}$, but the decoding error at $t=20$ is always much smaller than the center of mass decoding of the original signal. Finally, a major question motivating this study was to determine which network regime, in terms of inhibition strength, would be most suitable for decoding in such networks. The results of these studies are summarized in Figure 2C which shows the decoding improvement at different time steps for different inhibition parameters. Early on there is little dependence of the results on the strength of inhibition, but later there is some advantage for inhibition values around 0.06 . Interestingly, this is close to the transition region between the domain of decaying input and the domain of sustained activity. 

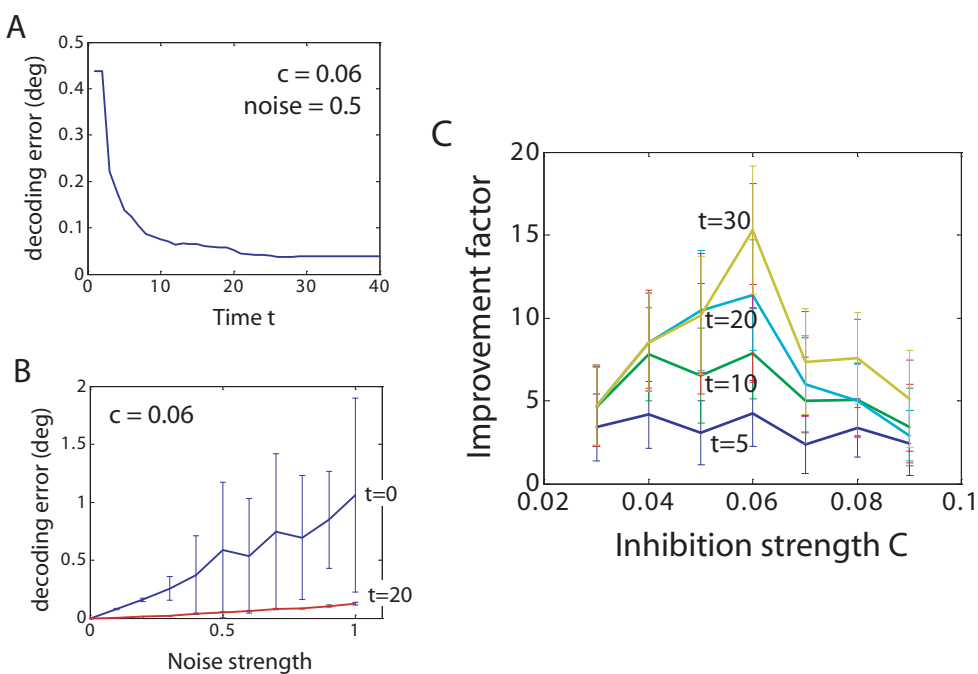

Fig. 2. Temporal aspects and decoding quality with different levels of inhibition

\section{Conclusion}

In this study we analyzed population decoding with recurrent networks with biologically motivated modifications. We thereby included sustained input, a simplified and non-global gain function, and different levels of inhibition. We found that population decoding with such competitive networks is very powerful even when continuously driven by external input. We also found that inhibition levels at the onset of the memory regime showed best decoding performances.

\section{References}

1. Zemel, R., Dayan, P., Pouget, A.: Probabilistic interpretation of population code. Neural Computation 10 (1998) 403-430

2. Pouget, A., Dayan, P., Zemel, R.: Information processing with population codes. Nature Reviews Neuroscience 1 (2000) 125-132

3. Pouget, A., Zhang, K., Deneve, S., Latham, P.: Statistically efficient estimation using population coding. Neural Computation 10(2) (1998) 373-401

4. Wu, S., Amari, S., Nakahara, H.: Population coding and decoding in a neural field: a computational study. Neural Computation 14 (2002) 999-1026

5. Wilson, H., Cowan, J.: A mathematical theory of the functional dynamics of cortical and thalamic nervous tissue. Kybernetik 13 (1973) 55-80

6. Grossberg, S.: Contour enhancement, short-term memory, and constancies in reverberating neural networks. Studies in Applied Mathematics 52 (1973) 217-257

7. Amari, S.: Dynamics of pattern formation in lateral-inhibition type neural fields. Biological Cybernetics 27 (1977) 77-87

8. Deneve, S., Pouget, A., Latham, P.: Divisive normalization, line attractor networks and ideal observers. Advances in Neural Information Processing Systems 11 (1999) 104-110 